\title{
TSUNAMI OF MOBILITY ELECTRONICS
}

Global projected sales data of passenger cars and light commercial vehicles will be about 100 mn plus units in 2018. Studies also indicate that with innovations in electronics and embedded systems, and with continued emphasis on energy efficiency, electronics has grown to $40 \%$ in conventional vehicles and to about $75 \%$ in XEVs. With the thrust on cost reduction and miniaturisation, several out of the box affordable semiconductor technologies that can survive $-40^{\circ}$ to $+85^{\circ} \mathrm{C}$ will propel the tsunami in mobility electronics and reduce product development cycles.

Further, the advancement in materials has increased power density of semiconductors and has enabled the reduction of size and weight, improved thermal dissipation, and made it possible to store enormous memory in tiny footprints.

The enormity of data storage capacity is causing radical paradigm shifts. The most obvious electronics is embedded across safety aspects, body applications cabin and underbody, in powertrain applications, and across XEVs. The penetration has been rapid, consumer friendly and in most of the cases, affordable. For the millennial digital travellers, the radical and incremental innovative applications will continue to be in the safety and comfort aspects, connectivity, prognostics and diagnostics, powertrains, and flexible vehicle platforms.

\section{FUTURE SOLUTIONS}

Some of the upcoming examples are biometric vehicle access and safety, retina scanners and finger prints, virtual power transfer to upsize or downsize engines, virtual transfers between energy storage devices, and driver over-ride systems to stop a vehicle in case of emergency or driver error. Telematics and vehicle tracking systems will become a necessity and regulators may use data from these to monitor erratic road behaviours and driving habits for insurance companies.

High density influx of Infotainment and Connectivity of vehicles will be a given, due to the availability of multiple networks and protocols, while on the roads. Regulators will mandate proactive prognostics and diagnostics for real-time personal health monitoring, breakdowns and accidents. In addition, deploying advanced lightweight recyclable advanced materials for flexible vehicle platforms will have consumers convert and adopt their vehicles for multiple usages and for multi-coloured exterior panels and interiors.

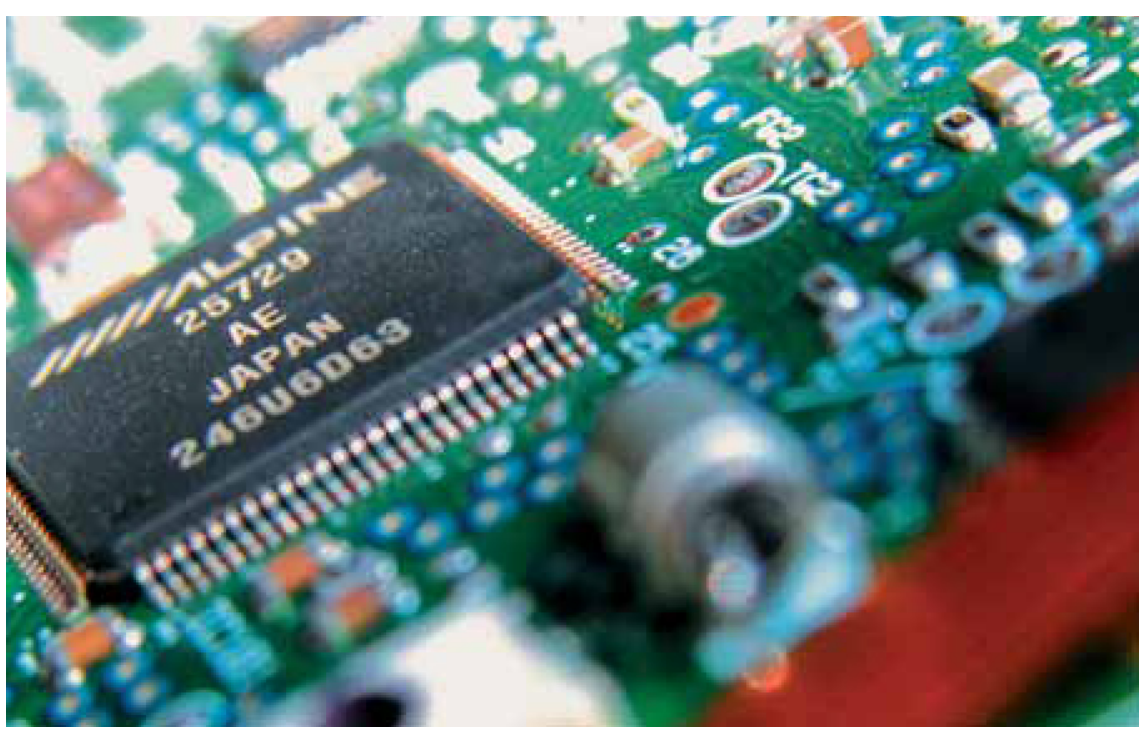

\section{PUSHING THE ENVELOPE}

The passion of mobility fraternity and excitement for consumers is that many of the mobility electronic technologies and features that have appeared to be science fiction will become mass market realities in about five years from now. The innovations in memory storage capacity and data analytics, and persistence of the digital-driven world for a safer and energy efficient mobility will continue to push the envelope for the growth of automotive electronics. 